\title{
Anti-inflammatory activity of berberine in non-alcoholic fatty liver disease via the Angptl2 pathway
}

\author{
Zengsheng Lu', Beihui He', Zhiyun Chen², Maoxiang Yan² and Liyan $\mathrm{Wu}^{3{ }^{*}}$ (D)
}

\begin{abstract}
Background: Nonalcoholic fatty liver disease (NAFLD) has become the most common liver disease worldwide. Recent studies have shown that the Angptl2 pathway mediated hepatic inflammatory response plays an important role in the progression of nonalcoholic fatty liver disease. Our study investigated the possible molecular mechanisms of berberine (BBR) in the treatment of the liver inflammatory response in the livers of rats with high-fat diet-induced NAFLD via the Angptl2 pathway.

Results: At the end of 12 weeks, compared with the control group rats, the high-fat- diet group rats showed obvious pathological and biochemical changes. The levels of pro-infalmmatory cytokines (CCL2, TNF-a) were increased, the infiltration of inflammatory cells (CCR2) was elevated, and the hepatic mRNA and protein levels of Angpt|2, NF-KB and Foxo1 were increased to different degrees. Nevertheless, following treatment with BBR, liver tissue pathology, biochemical data, and Angptl2 pathway-related genes expression were significantly ameliorated.

Conclusions: Our findings demonstrate that BBR might attenuate the liver inflammatory response in the livers of rats with high-fat diet-induced NAFLD through the regulation of the Angpt/2 pathway.
\end{abstract}

Keywords: Nonalcoholic fatty liver disease, Berberine, Angpt|2, Inflammatory response

\section{Background}

Nonalcoholic fatty liver disease (NAFLD), which includes nonalcoholic simple fatty liver, non-alcoholic steatohepatitis (NASH), liver fibrosis, cirrhosis, and hepatocellular carcinoma has become the most common liver disease worldwide, with a global incidence of approximately $24 \%$ [1]. The prevalence of adults with NAFLD in Shanghai and Guangdong Province, China, is approximately $15 \%[2,3]$, and the incidence rate is increasing each year. In addition, NAFLD also promotes the progression of other systemic diseases, such as cardiovascular diseases and type 2 diabetes, among others $[4,5]$. Nevertheless, the pathogenesis and clinical

\footnotetext{
*Correspondence: wuliyan0118@163.com

${ }^{3}$ Department of Gastroenterology, Tongde Hospital of Zhejiang Province, 234 Gucui Road, Zhejiang 310012, Hangzhou, China

Full list of author information is available at the end of the article
}

treatment of NAFLD have yet to be elucidated until now. Except lifestyle interventions, therapeutic approaches mainly include antioxidants (such as vitamin E) and peroxisome proliferator activated receptor agonists (such as thiazolidinediones) [6-8], but these interventions are associated with lack of organ or cell selectivity, and limited specificity, as well as side effects. Consequently, there is an urgent need to study new treatments for NAFLD. Recent studies have shown that metabolic syndrome consists of chronic, low-grade systemic inflammation, and NASH is considered to be the manifestation of metabolic syndrome in the liver [9]. Certain pro-inflammatory cytokines secreted by adipocytes and macrophages stimulate liver inflammatory responses and inflammatory cell infiltration in the liver by stimulating inflammatory signaling pathways, and participate in the development of NASH $[10,11]$. 
Berberine (BBR) is a kind of isoquinoline alkaloid isolated from the Chinese medicinal herb Rhizoma coptidis, which has been used in traditional Chinese medicine (TCM) for centuries. It is well known that BBR has many pharmacological properties with respect to metabolic diseases and many other inflammatory diseases $[12,13]$. Recently studies showed that BBR plays important roles in treating NAFLD, such as increasing insulin sensitivity, improving glucose and lipid metabolic disorders, regulating intestinal microbiota and alleviating oxidative stress; these findings suggest that BBR may serve as a potential drug for NAFLD [14-16]. However, studies on BBR treatment of the hepatic inflammatory response in NAFLD are still unclear. Angiopoietin-like protein 2 (Angptl2), a new secretory glycoprotein, belongs to the angiogenic-like protein family and is secreted by adipose tissue, macrophages (mainly Kuffer cells, KCs), and endothelial cells, among others [17]. Under normal conditions, Angptl2-mediated signal transduction contributes to angiogenesis and tissue damage repair [17], whereas excessive Angptl2 signaling leads to chronic inflammation, which is accompanied by obesity and metabolic syndrome [17], type 2 diabetes [18], atherosclerosis [19], and even certain tumors [20].Angptl2 activates Racl through integrins, which activates nuclear factor-kappaB (NF- $\mathrm{kB})$ and inhibits $\kappa \mathrm{B}$ inhibitor $(\mathrm{I} \kappa \mathrm{B})$, and promotes the release of inflammatory mediators, such as TNF- $\alpha$ and CCL2, and the aggregation of inflammatory cells; these processes, in turn, lead to the development of chronic inflammation of the liver. Based on these data, our study used a highfat diet-induced rat model of NAFLD to study whether BBR has an anti-NAFLD effect by inhibiting the hepatic inflammatory response via the Angptl2 pathway.

\section{Results}

BBR ameliorates hepatic steatosis and inflammation in HFD-fed rats

To confirm the therapeutic effect of BBR, we examined the effect of BBR on the liver of rats with HFD-fed induced NAFLD rats. As shown in Fig. 1, compared with those in the ND group, the liver tissues of rats in the HFD group showed obvious steatosis, inflammatory cell infiltration, and focal necrosis (Fig. 1a-c). Moreover, the NAFLD activity score (NAS) increased significantly (Fig. 1d). Compared with the HFD group, the HFD + BBR group showed a decrease in hepatic steatosis, inflammatory cell infiltration and NAS (Fig. 1).

\section{Biochemical changes in serum and liver in NAFLD rats} Serum ALT, AST, CHOL, FFAs, and liver TG and CHOL showed different degrees of increase (Fig. 2a-c and e-g). Compared with those in the HFD group, serum ALT, AST, CHOL, FFAs, hepatic TG, and hepatic CHOL in the HFD + BBR group decreased to 35.02, 22.20, 24.63, 15.74, 16.65 and $31.25 \%$, respectively (Fig. 2a-c and e-g).
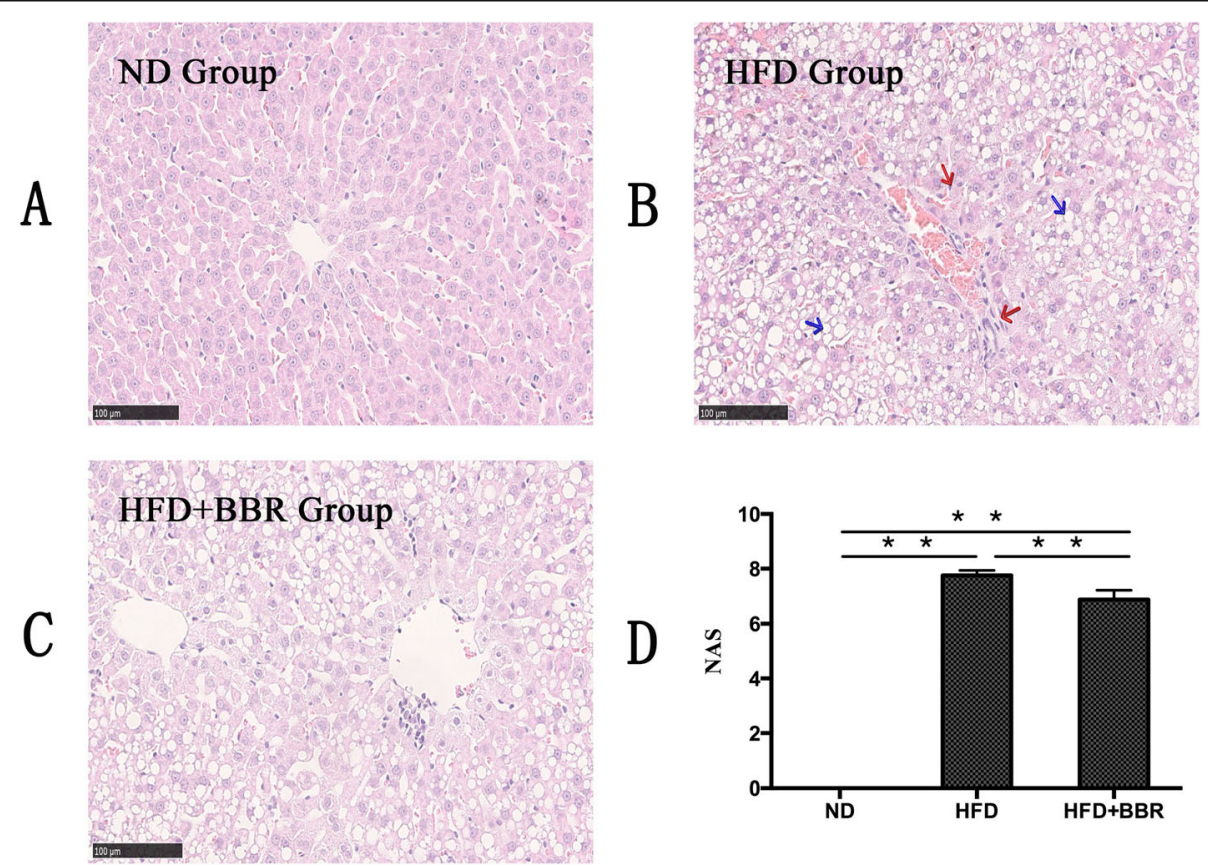

Fig. 1 BBR ameliorates hepatic steatosis in HFD-fed rats. The liver isolated from rats fed with a normal diet (ND), high-fat diet (HFD) or high-fat plus Berberine (HFD + BBR) were stain with hematoxylin and eosin. a ND group; (b) HFD group; (c) HFD + BBR group. Photographs are at $100 \times$ magnification. Steatosis shown in blue arrow, necrosis and inflamation shown in red arrows. $\mathbf{d}$ NAFLD activity score. Data are presented as mean $\pm \mathrm{SD}$ from eight rats. ${ }^{* *} P<0.01, * 0<0.05 . n=8 /$ group 


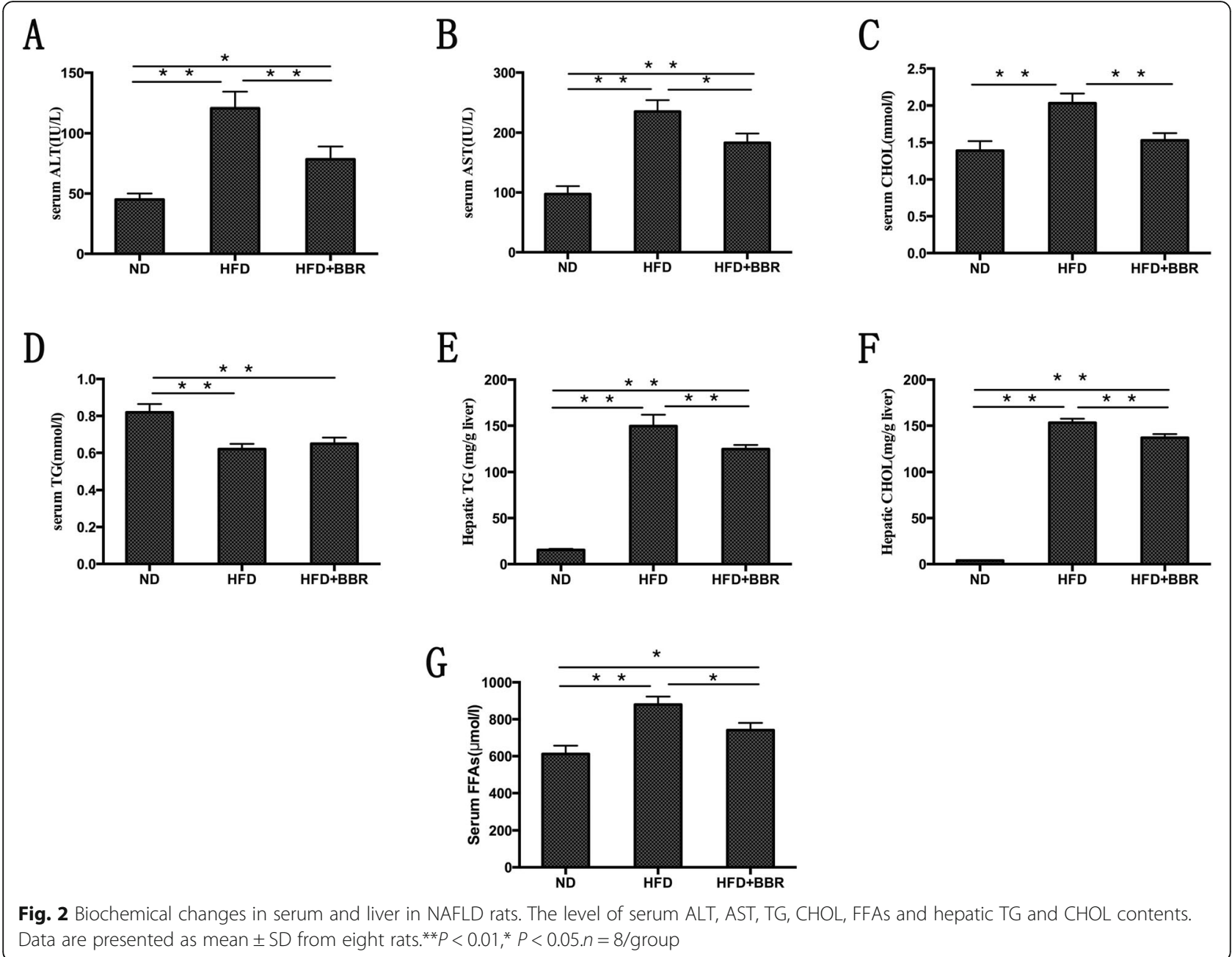

Interestingly, serum TG in the HFD group showed a decrease compared with the ND group, and after the intervention with BBR, serum TG was increased (Fig. 2d).

\section{Serum cytokines in NAFLD rats}

To verify the therapeutic effect of BBR, we examined the effect of BBR on the serum cytokines in rats with HFD- induced NAFLD. As shown in Fig. 3, compared with those in the ND group, the serum CCL2 and TNF- $\alpha$ levels were significantly increased in the HFD group $(P<0.01)$. After the intervention with BBR, the serum CCL2 and TNF- $\alpha$ levels in the HFD + BBR group were significantly decreased compared with the HFD group $(P<0.05)$.
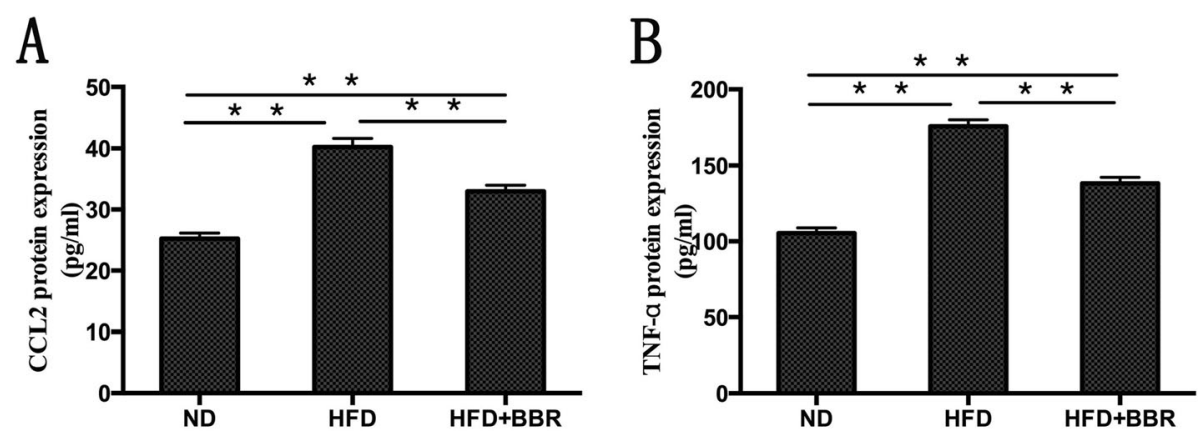

Fig. 3 Serum proinflammatory cytokines in NAFLD rats, which were measured by ELISA. a serum CCL2 levels; (b) serum TNF-a levels. Data are presented as mean $\pm \mathrm{SD}$ from eight rats. ${ }^{*} P<0.01,{ }^{*} P<0.05 . \mathrm{n}=8 /$ group 
Angptl2 pathway-related mRNA expression in the liver tissues of NAFLD rats

The levels of Angptl2, Foxo1, CCR2 and NF- $\mathrm{B}$ mRNA in the HFD group showed different degrees of increase (Fig. 4). However, the levels of Angptl2, Foxo1, CCR2 and NF- $\mathrm{kB}$ mRNA in the HFD + BBR group were significantly secreased at 12 weeks compared with those in the HFD group (Fig. 4).

\section{Angpt|2 pathway-related protein expression in the liver tissues of NAFLD rats}

We examined the protein levels of Angptl2, Foxo1, NF$\kappa \mathrm{B}, \mathrm{p}-\mathrm{NF}-\kappa \mathrm{B}$ and CCR2 by using Western blot analysis. As shown in Fig. 5b, $c$, e and $f$, the protein levels of Angptl2, Foxo1, p-NF- $\mathrm{kB}$ and CCR2 were significantly increased compared with the those in ND group. An increasing trend in the liver protein levels of NF- $\mathrm{KB}$ was observed in the HFD group, although no statistically significant difference was observed between the HFD group and ND group (Fig. 5d). After the intervention with $\mathrm{BBR}$, we found that the protein levels of Angptl2, P-NF$\mathrm{KB}$ and $\mathrm{CCR} 2$ showed remarkable decreases compared with those in HFD group (Fig. 5b, e and f). We also observed a decrease in the liver protein levels of Foxo1 and NF- $\kappa B$ in the HFD + BBR group compared with those in the HFD group, but these trends were not significant (Fig. 5c and d).

\section{Discussion}

Approximately 25\% of patients with NAFLD have NASH, which is characterized by inflammation, hepatocyte ballooning, and hepatocyte injury [21]. The pathogenesis of NASH has not been clearly eluciated. The "two-hits" theory proposed by Day et al. is widely accepted by the majority scholars [22]. The first hit is "fatty degeneration", in which lipotoxicity-induced mitochondrial dysfunction and hepatocyte steatosis make the liver more prone to inflammation under the action of the second hit. The second hit is enhancement of liver lipid peroxidation and an increase of FFAs. Although this theory does not explain all of the problems, it can be surmised that chronic inflammation plays an important role in the progression of $\mathrm{NASH}$, and thus reducing the liver inflammatory response may be a potential target for treatment of NASH.

Adipocyte, macrophage-derived Angptl2 is a key mediator linking obesity and the related metabolic diseases to the inflammatory response in adipose tissues [17]. Several recent studies have shown that high-fat diet-induced obesity and the related metabolic diseases in the visceral adipose tissue of mice express a large amount of Angptl2, which significantly promotes macrophage aggregation and proinflammatory cytokine expression [23], leading to chronic inflammation and systemic insulin resistance of adipose tissue [24].AP2-Angpt12 mice (adipose tissue- specific promoter Angptl2, AP2-Angpt12) showed a marked increase in inflammatory cytokines (CCL2, and TNF- $\alpha$ ) and inflammatory cell surface markers (CCR2) in adipose tissue [17]. In mice injected with Angptl2 by adenovirus, it was found that the accumulation of liver lipids and the expression of genes involved in fatty acid synthesis and metabolism were significantly increased, which in turn contributed to the
A

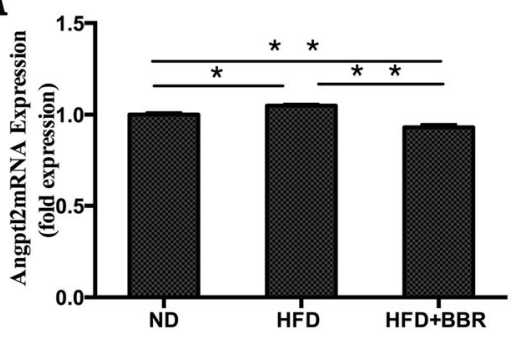

C

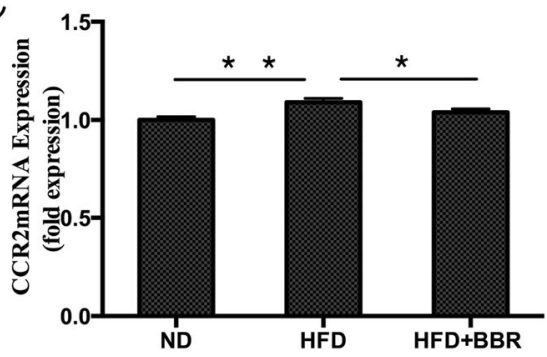

B

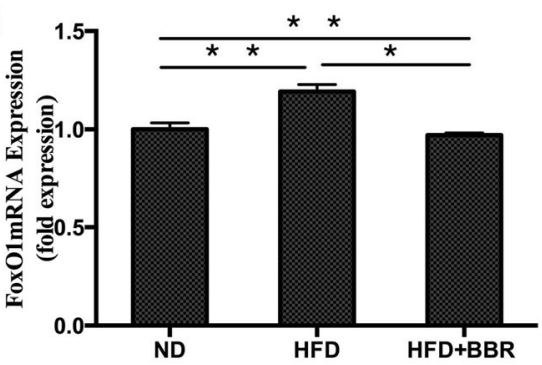

D

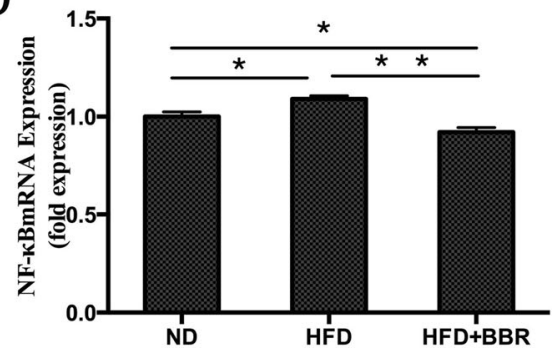

Fig. 4 Levels of Angptl2, Foxo1, CCR2 and NF-KB-mRNA were measured by real time-PCR and normalized by GAPDH expression; ${ }^{*} P<0.01,{ }^{*} P<0.05 . n=8 /$ group 


\section{$\begin{array}{lll}1 & 2 & 3\end{array}$}

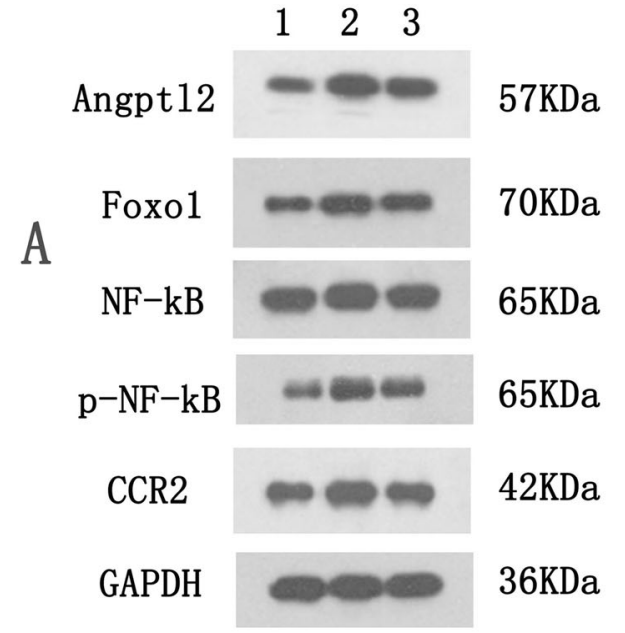

B

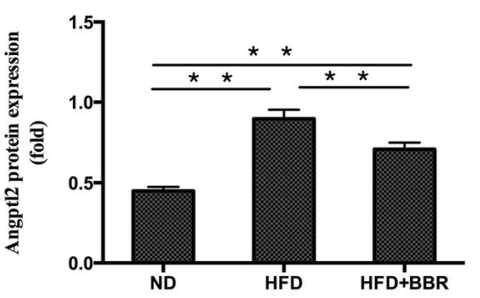

C

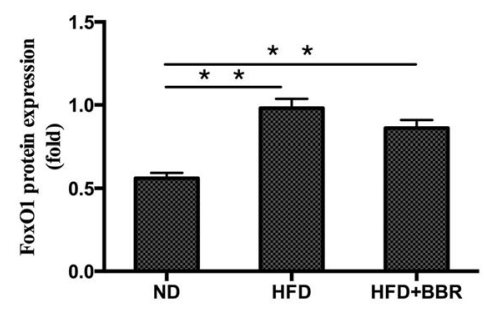

D

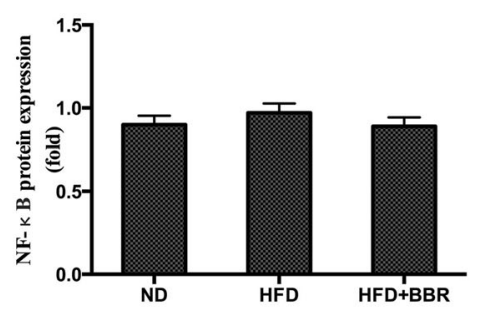

$\mathrm{E}$

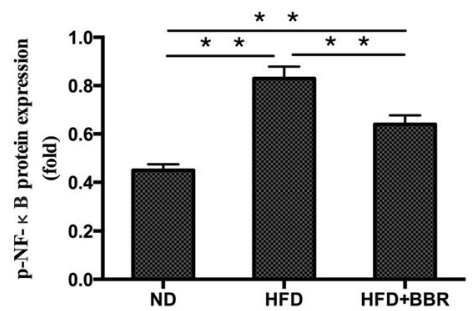

F

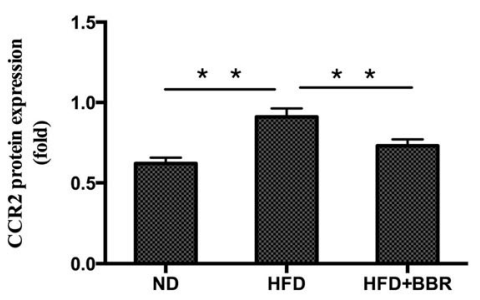

Fig. 5 The protein levels of Angptl2, Foxo1, NF-KB, p-NF-KB and CCR2 were measured by Western blot analysis and normalized by GAPDH expression. Data are presented as mean \pm SD from eight rats. ${ }^{*} P<0.01$, $P<0.05 . n=8 /$ group

development of fatty liver [25]. The infiltration of inflammatory cells and the chronic inflammation of adipose tissue induced by a high-fat diet in Angptl2knockout mice were significantly reduced compared with those in wild-type mice, and glucose tolerance and insulin sensitivity were significantly increased [17]. Our study showed that the rats with high-fat diet-induce NASH were characterized by significant steatosis, inflammatory cell infiltration and necrosis. In addition, serum transaminase, lipid levels, liver lipid levels, and NAS showed different degrees of increase. Furthermore, we found that the Angptl2 expression level in the liver tissue of the HFD group was significantly higher than that in the ND group. The inflammatory cascade involved the activation of NF- $\mathrm{kB}$, the numbers of CCR2+ inflammatory cells in liver tissue increased, and proinflammatory cytokines, such as CCL2, and TNF- $\alpha$, in the peripheral blood were significantly elevated. Therefore, according to the above results, we speculate that the Angptl2-mediated signaling pathway is involved in the development of the NASH liver inflammatory response.

However, after BBR intervention, the pathology of liver tissue in NASH rats was significantly improved, and serum transaminases, lipid levels, liver lipids and NAS were significantly ameliorated. In addition, we found that the expression level of Angptl2 in the liver tissue of the HFD + BBR group decreased, the phosphorylation level of NF- $\mathrm{KB}$ decreased, the numbers of CCR2+ inflammatory cells in the liver tissue decreased, and the proinflammatory cytokines, such as CCL2 and TNF- $\alpha$, in 
the peripheral blood significantly decreased. Previous studies by other scholars have shown that BBR ameliorate NAFLD by reducing endoplasmic reticulum stress and regulating the hepatic sirtuin 1-uncoupling protein 2 and AMPK pathways [26-28]. These findings indicated that BBR may ameliorate the liver inflammatory response in NASH through the Angptl2-mediated signaling pathway.

The Angptl2 mediated signal transduction pathway, in addition for directly activating downstream inflammatory cascades and releasing inflammatory mediators, may also be involved in regulating the following mechanisms to regulate the liver inflammatory responses of NASH: (1) Foxo1 is a transcription factor in the forkhead box proteins (FOX) family that negatively regulated by insulin signaling and regulates other genes to control glucose and lipid metabolism [29], oxidative stress and apoptosis in the liver [30].Foxo1 binding to the region-specific insulin response element enhancer (nt-1457, nt-1258) of the Angptl2 promoter, upregulates the expression of Angptl2 in adipocytes through the Foxo1 pathway, promotes the release of inflammatory cytokines, such as CCL2, and participates in inflammation and systemic insulin resistance [23]. It has been found that the activity and expression of Foxo1 in liver are upregulated in NASH patients, from simple fatty liver to nonalcoholic steatohepatitis [31]. Interestingly, Foxo1 knockout significantly reduces the Angptl2 mRNA expression [23]. Our study results are consistent with the literature reports. Foxo1 expression in the HFD group was significantly higher than that in the ND group. However, after BBR intervention, the Foxo1 expression level was significantly decreased. (2) TNF- $\alpha$ upregulates Angptl2 expression through the Foxo1 pathway, further promoting inflammatory mediators expression and insulin resistance occurence [23].Nevertheless, lentiviral-mediated siRNA interference with the Foxo1 gene can inhibit the transcriptional activity of the Angptl2 promoter, and reduce the adipocyte Angptl2 expression induced by TNF- $\alpha$ [32]. It can be observed that the above mechanisms can activate the inflammatory cascade mediated by the Angptl2 pathway alone or in combination, which ultimately leads to the progression of NASH liver inflammation, and BBR may ameliorate the liver inflammatory response in NASH by affecting the Angptl2 signaling pathway.

In conclusion, the findings of this study show that the change in the Angptl2 signaling pathway is one of the mechanisms of the hepatic inflammatory response induced by a high-fat diet in NASH rats, and BBR may exert anti-inflammatory effects by regulating the Angptl2 signaling pathway. However, the in vitro experiments and specific mechanisms of BBR treatment of NASH liver inflammation require further study.

\section{Conclusions}

Hepatic inflammatory response is a key rate-limiting step in the progression of nonalcoholic simple fatty liver to NASH. Angptl2 signaling pathway activation plays an important role in the liver inflammatory response induced by a high-fat diet in NASH rats. BBR may exert an anti-inflammatory effect through regulation of the Angptl2 signaling pathway. The study results offer insight into the pharmacological mechanisms of BBR in the treatment of NAFLD.

\section{Methods}

\section{Animal experiments}

A total of 24 male SD rats (age 6 weeks, each weighing 180-200 g) were purchased from Shanghai Sipper-BK Laboratory Animal Co. Ltd. (production license: SCXK (Hu)2013-0016). All rats were randomly divided into three groups after 1 week of adaptive feeding as follows: (i) control group (ND group,8 rats), which was normally fed with feedstuff (21.5\% protein, $12.3 \%$ fat, and $66.2 \%$ carbohydrate) produced by Jiangsu Synergic Bioengineering Co., Ltd.; (ii) high-fat diet group (HFD group, 8 rats) was given high-fat diet (comprising 21.6\% protein, $36.1 \%$ fat, $42.3 \%$ carbohydrate; $82.75 \%$ basal diet $+10 \%$ lard, $2 \%$ cholesterol, $0.25 \%$ bile salt, and $5 \%$ egg yolk powder; feedstuff was produced by Jiangsu Synergic Bioengineering Co., Ltd); (iii) HFD + BBR group (8 rats) was fed with high-fat diet for 4 weeks, and intragastric administration of BBR at $300 \mathrm{mg} \cdot \mathrm{kg}-1 \cdot \mathrm{d}-1$ was performed for 8 weeks starting from week 5 [Berberine (HPLC grade, $\geq 98 \%$ ) were purchased from Chengdu Herbpurify Co., Ltd. Sichuan, China]. The normal and the model groups were administered with an equal volume of $0.9 \%$ sodium chloride, exposed to continuous light and 12:12 $\mathrm{h}$ light/dark cycle, and provided with food and water ad libitum. All rats were sacrificed at the end of week 12, and were fasted on water for $12 \mathrm{~h}$ the night before treatment. Under anesthesia, blood samples were extracted intraocularly, and liver specimens were collected. The right liver lobe was extracted and fixed in 10\% neutral formaldehyde for pathological observation, and the other liver tissues were stored at $-80^{\circ} \mathrm{C}$ for later use. All experimental animals were anesthetized by intraperitoneal injection with sodium pentobarbital $(60 \mathrm{mg} / \mathrm{kg})$. After deep anesthesia, cervical dislocation was performed and euthanasia was performed. Animal studies were approved by the Animal Use and Care Committee of Tongde Hospital of Zhejiang Province, and were conducted in accordance with the National Research Council Guide for Care and Use of Laboratory Animals.

\section{Serum biochemistry and cytokines of rats with NAFLD}

Triglyceride (TG), and Cholesterol (CHOL) were used to measure the TG (Shanghai DESAY Diagnostic 
Table 1 The real time-PCR primers for the validated genes

\begin{tabular}{lll}
\hline Gene & & primers \\
\hline Angpt12 & forward & 5'- GCA GGA GAG AAG AGG TाT CAA G - 3' \\
& reverse & 5'- TCC AAG CCA CCA GTA AGT CAT A - 3' \\
Foxo1 & forward & 5'- CAG GCC GGA GTT TAA CCA GT - 3' \\
& reverse & 5'- CTC GCT CTC TTC TAG CAG GC -3' \\
CCR2 & forward & 5'- ACC CTG TाT CGC TGT AGG ATT -3' \\
& reverse & 5'- AAG TGC ATG TCA ACC ACA CAG -3' \\
NF-KB & forward & 5'- GGC CTC ATC CAC ATG AAC T -3' \\
& reverse & 5'- TAA TGG CTT GCT CCA GGT CT -3' \\
GAPDH & forward & 5'- GAC AAC TाT GGC ATC GTG GA -3' \\
& reverse & 5'- ATG CAG GGA TGA TGT TCT GG -3'
\end{tabular}

Technology Co., Ltd., and batch number 571/044/1), and CHOL (Shanghai DESAY Diagnostic Technology Co., Ltd., and batch number $130 / 036 / 2$ ) content in serum as well as liver tissues, according to the kit instructions. Levels of serum alanine transaminase (ALT) and glutamic-oxalacetic transaminase (AST) were measured according to the kit instructions for ALT (Shanghai DESAY Diagnostic Technology Co., Ltd., and batch number 27012/972006/1) and AST (Shanghai DESAY Diagnostic Technology Co., Ltd., and batch number 26010/ 962004/1) tests. Levels of serum free fatty acids (FFAs) measured according to the kit instructions for FFAs (R\&D system Co., Ltd., and batch number CK-E30208R) tests. Levels of serum cytokines, including $\mathrm{C}-\mathrm{C}$ chemokine ligand 2 [CCL2, also called monocyte chemo attractant protein, MCP-1,(Shanghai westang bio-tech Co., LTD, and batch number F3760)], Tumour necrosis factor- $\alpha$ [TNF- $\alpha$ (Shanghai westang bio-tech Co., LTD, and batch number MM-0180R1)], were measured by ELISA (Bio-Ter ELX800, USA).

\section{Histological examination}

The liver tissues were fixed with $10 \%$ neutral formaldehyde, and embedded in paraffin after ethanol dehydration. Subsequently, tissues were cut into 5- $\mu \mathrm{m}$-thick sections, and monitored for liver steatosis and inflammation using $\mathrm{H}$ \& $\mathrm{E}$ (hematoxylin and eosin) staining optical microscopy. The diagnostic criteria were based on the European guidelines for the diagnosis and treatment of NAFLD [33].

\section{Real time-PCR}

Trizol reagent was used to extract the total RNA in liver tissues of the 24 rats. PrimeScript kit (Takara, Tokyo, Japan) was used to synthesize cDNA by reverse transcription in a $20 \mu \mathrm{L}$ system. The total RNA was placed in an automatic water bath at $37^{\circ} \mathrm{C}$ for $15 \mathrm{~min}$, and $85^{\circ} \mathrm{C}$ for $30 \mathrm{~s}$ according to the instructions. RT-PCR was performed by ABI 7900 PCR instrument and SYBR Premix EX Taq (Takara, Tokyo, Japan). The $20 \mu \mathrm{L}$ of PCR reaction system contained $2 \mu \mathrm{L}$ cDNA sample, $0.4 \mu \mathrm{L}$ $(10 \mu \mathrm{M})$ specific upstream and downstream primers, and $10 \mu \mathrm{L}$ SYBR Premix EX TaqII (TliRNaseH Plus) $(2 \times)$, $0.4 \mu \mathrm{L}$ ROX Reference Dye $(50 \times)$, as well as $6.8 \mu \mathrm{L}$ RNase-free $d_{d d} \mathrm{H}_{2} \mathrm{O}$. RT-PCR primers were synthesized by Sangon Biotech (Shanghai) Co., Ltd. (Table 1.). The PCR reaction conditions involved denaturation at $95^{\circ} \mathrm{C}$ for $3 \mathrm{~min}, 95^{\circ} \mathrm{C}$ for $5 \mathrm{~s}$, and $60^{\circ} \mathrm{C}$ for $45 \mathrm{~s}$, respectively. The PCR included 40 cycles of amplification, and each sample had three wells, with GAPDH as internal reference. Furthermore, $2^{-\Delta \Delta \mathrm{Ct}}$ was used to calculate the relative gene expression.

\section{Western blot analysis}

Total protein was exacted from liver tissue by using Lysis buffer containing phosphatase inhibitors (100 $\mathrm{mM})$, protein enzyme inhibitor $(1000 \mathrm{mM})$ and PMSF $(100 \mathrm{mM})$. Protein concentrations were determined using a BCA protein quantitative kit (Applygen Co., Ltd., China). The proteins were electrophoreses in $10 \%$ sodium dodecyl sulfate-polyacrylamide gels and transferred onto polyvinylidene difluoride membranes. The membranes were blocked with $5 \%$ bovine serum albu$\min$ for $1 \mathrm{~h}$. The memebrane were the incubated at $4{ }^{\circ} \mathrm{C}$ overnight with primary antibodies against Angptl2, Foxo1, NF-кB, p-NF-kB, CCR2 and GAPDH (information of these antibodies are shown in Table 2). The membranes were washed and incubated with

Table $\mathbf{2}$ Information of antibodies used for western blots

\begin{tabular}{llll}
\hline Antibody name & Catalog number & Vendor & Working concentration \\
\hline Angptl2 & $12,316-1-$ AP & Affinity Biosciences & $1: 500$ \\
Foxo1 & DF7110 & Affinity Biosciences & $1: 1000$ \\
NF-KB & AF5006 & Affinity Biosciences & $1: 1000$ \\
p-NF-KB & Ab3266 & Affinity Biosciences & $1: 1000$ \\
CCR2 & DF2711 & Affinity Biosciences & $1: 1000$ \\
GAPDH & $60,004-1-\mathrm{lg}$ & Proteintech Group & $1: 5000$ \\
\hline
\end{tabular}


horseradish peroxidase-conjugated immunoglobulin $\mathrm{G}$ antibody (Proteintech Group, China) at $37^{\circ} \mathrm{C}$ for $1 \mathrm{~h}$. Signals were detected through the chemiluminescent reaction by using a gel imaging system (FCE ProteinSimple chemiluminescent imaging system, ProteinSimple, USA). The relative expression levels of the target proteins were normalized against that of the GAPDH protein.

\section{Statistical analysis}

All data were analyzed using SPSS17.0 software (SPAA Inc., USA). Results were expressed as mean \pm standard deviation and one-way analysis of variance was used. Comparison among groups used LSD analysis (homogeneity of variance) or Dunnett's T3 (heterogeneous variance). $P<0.05$ was statistically significant.

\section{Abbreviations}

NAFLD: Nonalcoholic fatty liver disease; NASH: Non-alcoholic steatohepatitis; BBR: Berberine; Angptl2: Angiopoietin-like protein 2; KCs: Kuffer's cell; TG: Triglyceride; CHOL: Cholesterol; ALT: Alanine transaminase; AST: Glutamicoxalacetic transaminase; FFAs: Free fatty acids; CCL2: C-C chemokine ligand 2: TNF-a: Tumour necrosis factor-a; NAS: NAFLD activity score

\section{Acknowledgements}

Not applicable.

\section{Authors' contributions}

LW designed the experiment and revised the manuscript. ZLdrafted the article and analyzed the data. ZC revised it critically for important intellectual content. BH, LW and MY performed the experiment. All authors read and approved the final manuscript.

\section{Funding}

The work was supported by the Scientific Research Fundation of State Administration of Traditional Chinese Medicine of Zhejiang Province (2016ZB004), the Scientific Research Fundation of State Administration of Traditional Chinese Medicine of Zhejiang Province (2019ZA024), the Medical Health Science and Technology Project of Zhejiang Provincial Health Commission (2018KY322), and the Program of Zhejiang Province Natural Science Foundation (LY17H290007). The funder had no role in study design, data collection and analysis, decision to publish, or preparation of the manuscript.

\section{Availability of data and materials}

The datasets used and /or analysed during the current study available from the corresponding author on reasonable request.

\section{Ethics approval and consent to participate}

The experimental protocols were established according to the Declaration of Helsinki, and the experiments were approved by the Experimental Animal Ethics Committee of the Animal Use and Care Committee of Tongde Hospital of Zhejiang Provice, (Approval NO::[2019]013).

\section{Consent for publication}

Not applicable.

\section{Competing interests}

The authors declare that they have no competing interests.

\section{Author details}

'Department of Infectious Diseases, Tongde Hospital of Zhejiang Province, 234 Gucui Road, Zhejiang 310012, Hangzhou, China. ${ }^{2}$ Key Laboratory of Diagnosis and Treatment of Circulatory Diseases in Combination with Traditional Chinese and Western Medicine of Zhejiang Province, Zhejiang Hospital of Traditional Chinese Medicine, 54 Youdian Road, Zhejiang 310006, Hangzhou, China. ${ }^{3}$ Department of Gastroenterology, Tongde Hospital of Zhejiang Province, 234 Gucui Road, Zhejiang 310012, Hangzhou, China.
Received: 4 July 2019 Accepted: 12 May 2020

Published online: 19 May 2020

\section{References}

1. Bedossa P. Pathology of non-alcoholic fatty liver disease. Liver Int. 2017; 37(Suppl 1):85-9.

2. Younossi Z, Anstee QM, Marietti M, Hardy T, Henry L, Eslam M, et al. Global burden of NAFLD and NASH: trends, predictions, risk factors and prevention. Nat Rev Gastroenterol Hepatol. 2018;15:11-20.

3. Fan JG, Farrell GC. Epidemiology of non-alcoholic fatty liver disease in China. J Hepatol. 2009:50:204-10.

4. Perry RJ, Samuel VT, Petersen KF, Shulman GI. The role of hepatic lipids in hepatic insulin resistance and type 2 diabetes. Nature. 2014;510:84-91.

5. Motamed N, Rabiee B, Poustchi H, Dehestani B, Hemasi GR, Khonsari MR, et al. Non-alcoholic fatty liver disease (NAFLD) and 10-year risk of cardiovascular diseases. Clin Res Hepatol Gastroenterol. 2017;41:31-8.

6. Basu P, Shah NJ, Farhat S, Siriki R, Mittimanj K, Atluri S, et al. Curcumin, antioxidant, and pioglitazone therapy with inclusion of vitamin $E$ in non alcoholic fatty liver disease-a randomized open label placebo controlled clinical prospective trial (captive). Gut. 2013;62:A23.

7. Chalasani NP, Sanyal AJ, Kowdley KV, Robuck PR, Hoofnagle J, Kleiner DE, et al. Pioglitazone versus vitamin $E$ versus placebo for the treatment of nondiabetic patients with non-alcoholic steatohepatitis: PIVENS trial design. Contemp Clin Trials. 2009:30:88-96.

8. Haidarlo AJ, Rashidbeygi M, Ehsanbakhsh S. Vitamin E, pioglitazone and diet therapy for patients with nonalcoholic fatty liver disease (Nafld): evaluation of treatment. J Hepatol. 2012;56:S507.

9. Lasselin J, Capuron L. Chronic low-grade inflammation in metabolic disorders: relevance for behavioral symptoms. Neuroimmunomodulation. 2014:21:95-101.

10. Kanda H, Tateya S, Tamori Y, Kotani K, Hiasa K, Kitazawa R, et al. MCP-1 contributes to macrophage infiltration into adipose tissue, insulin resistance, and hepatic steatosis in obesity. J Clin Investig. 2006:116:1494-505.

11. Weisberg SP, Hunter D, Huber R, Lemieux J, Slaymaker S, Vaddi K, et al. CCR2 modulates inflammatory and metabolic effects of high-fat feeding. J Clin Investig. 2006;116:115-24.

12. Vuddanda PR, Chakraborty S, Singh S. Berberine: a potential phytochemical with multispectrum therapeutic activities. Expert Opin Investig Drugs. 2010; 19:1297-307.

13. Zhang J, Cao H, Zhang B, Cao H, Xu X, Ruan H, et al. Berberine potently attenuates intestinal polyps growth in ApcMin mice and familial adenomatous polyposis patients through inhibition of Wnt signalling. J Cell Mol Med. 2013;17:1484-93.

14. Liu $Y$, Zhang $L$, Song $H$, Ji G. Update on berberine in nonalcoholic fatty liver disease. Evid Based Complement Alternat Med. 2013;2013:308134

15. Yuan X, Wang J, Tang X, Li Y, Xia P, Gao X, et al. Berberine ameliorates nonalcoholic fatty liver disease by a global modulation of hepatic mRNA and IncRNA expression profiles. J Transl Med. 2015:13:24.

16. Zhu X, Bian H, Gao X. The potential mechanisms of Berberine in the treatment of nonalcoholic fatty liver disease. Molecules. 2016;21:10.

17. Tabata M, Kadomatsu T, Fukuhara S, Miyata K, Ito Y, Endo M, et al. Angiopoietin- like protein 2 promotes chronic adipose tissue inflammation and obesity- related systemic insulin resistance. Cell Metab. 2009;10:178-88.

18. Odagiri H, Kadomatsu T, Endo M, Masuda T, Morioka MS, Fukuhara S, et al. The secreted protein ANGPTL2 promotes metastasis of osteosarcoma cells through integrin alpha5beta1, p38 MAPK, and matrix metalloproteinases. Sci Signal. 2014;7:ra7.

19. Horio E, Kadomatsu T, Miyata K, Arai Y, Hosokawa K, Doi Y, et al. Role of endothelial cell-derived angptl 2 in vascular inflammation leading to endothelial dysfunction and atherosclerosis progression. Arterioscler Thromb Vasc Biol. 2014:34:790-800.

20. Aoi J, Endo M, Kadomatsu T, Miyata K, Nakano M, Horiguchi H, et al. Angiopoietin-like protein 2 is an important facilitator of inflammatory carcinogenesis and metastasis. Cancer Res. 2011;71:7502-12.

21. Michelotti GA, Machado MV, Diehl AM. NAFLD, NASH and liver cancer. Nat Rev Gastroenterol Hepatol. 2013;10:656-65.

22. Day CP, James OFW. Steatohepatitis: a tale of two "hits"? Gastroenterology. 1998;114:842-5

23. Zheng JY, Zou JJ, Wang WZ, Feng XY, Shi YY, Zhao Y, et al. Tumor necrosis factor-alpha increases angiopoietin-like protein 2 gene expression by activating Foxo1 in 3T3-L1 adipocytes. Mol Cell Endocrinol. 2011;339:120-9. 
24. Kadomatsu T, Endo M, Miyata K, Oike Y. Diverse roles of ANGPTL2 in physiology and pathophysiology. Trends Endocrinol Metab. 2014;25:245-54

25. Sasaki Y, Ohta M, Desai D, Figueiredo JL, Whelan MC, Sugano T, et al. Angiopoietin like protein 2 (ANGPTL2) promotes adipose tissue macrophage and T lymphocyte accumulation and leads to insulin resistance. PLoS One. 2015;10:e0131176.

26. Hotamisligil GS. Endoplasmic reticulum stress and the inflammatory basis of metabolic disease. Cell. 2010;140:900-17.

27. Xing $L$, Zhang $L$, Liu T, Hua YQ, Zheng PY, Ji G. Berberine reducing insulin resistance by up-regulating IRS-2 mRNA expression in nonalcoholic fatty liver disease (NAFLD) rat liver. Eur J Pharmacol. 2011;668:467-71.

28. Turner N, Li JY, Gosby A, To SW, Cheng Z, Miyoshi H, et al. Berberine and its more biologically available derivative, dihydroberberine, inhibit mitochondrial respiratory complex I: a mechanism for the action of berberine to activate AMP-activated protein kinase and improve insulin action. Diabetes. 2008;57:1414-8.

29. Cook JR, Matsumoto M, Banks AS, Kitamura T, Tsuchiya K, Accili D, et al. A mutant allele encoding DNA binding-deficient FoxO1 differentially regulates hepatic glucose and lipid metabolism. Diabetes. 2015;64:1951-65.

30. Arden KC. FOXO animal models reveal a variety of diverse roles for FOXO transcription factors. Oncogene. 2008;27:2345-50.

31. Valenti L, Rametta R, Dongiovanni P, Maggioni M, Fracanzan AL, Zappa M, et al. Increased expression and activity of the transcription factor FOXO1 in nonalcoholic steatohepatitis. Diabetes. 2008;57:1355-62.

32. Su D, Coudriet GM, Hyun Kim D, Lu Y, Perdomo G, Qu S, et al. FoxO1 links insulin resistance to proinflammatory cytokine IL-1 beta production in macrophages. Diabetes. 2009;58:2624-33.

33. Chalasani N, Younossi Z, Lavine JE, Diehl AM, Brunt EM, Cusi K, et al. The diagnosis and management of non-alcoholic fatty liver disease: practice guideline by the American Association for the Study of Liver Diseases, American College of Gastroenterology, and the American Gastroenterological Association (vol 107, pg 811, 2012). Am J Gastroenterol. 2012;107:1598.

\section{Publisher's Note}

Springer Nature remains neutral with regard to jurisdictional claims in published maps and institutional affiliations.

Ready to submit your research? Choose BMC and benefit from:

- fast, convenient online submission

- thorough peer review by experienced researchers in your field

- rapid publication on acceptance

- support for research data, including large and complex data types

- gold Open Access which fosters wider collaboration and increased citations

- maximum visibility for your research: over $100 \mathrm{M}$ website views per year

At $\mathrm{BMC}$, research is always in progress.

Learn more biomedcentral.com/submissions 